\title{
Modeling Investment Trends: A Logarithmic-Modified Markov Chain Approach
}

\author{
Imoh Udo Moffat ${ }^{1,(1)}$, James Augustine Ukpabio ${ }^{1}$, Emmanuel Alphonsus Akpan ${ }^{2,3, *,[(])}$ \\ ${ }^{1}$ Department of Statistics, University of Uyo, Uyo, Nigeria \\ ${ }^{2}$ Department of Mathematical Science, Abubakar Tafawa Balewa University, Bauchi, Nigeria \\ ${ }^{3}$ Federal School of Medical Laboratory Technology (Science), Jos, Nigeria
}

A R T CLE IN F O
Article History
Received 01 June 2020
Accepted 23 Sep 2020
Keywords
Convergence
Heteroscdasticity
Logarithmic transformation
Markov chain
Stochastic process
Transition matrix

\begin{abstract}
The study aimed at stabilizing the changing variance using the logarithmic transformation to achieve a significant proportion of stability and a faster rate of convergence of the steady state transition probability in Markov chains. The traditional Markov chain and logarithmic-modified Markov chain were considered. On exploring the yearly data on the stock prices from 2015 to 2018 as obtained from the Nigerian Stock Exchange, it was found that the steady state of logarithmic-modified Markov chain converged faster than the tradition Markov chain with efficiency in tracking the correct cycles where the stock movements are trending irrespective of which cycle it starts at time zero with differences in probability values by $1.1 \%, 0.7 \%,-0.41 \%$ and $-1.37 \%$ for accumulation, markup, distribution and mark-down cycles, respectively. Thus, it could be deduced that the logarithmic modification enhances the ability of the Markov chain to tract the variation of the steady state probabilities faster than the traditional counterpart.
\end{abstract}

(c) 2020 The Authors. Published by Atlantis Press B.V. This is an open access article distributed under the CC BY-NC 4.0 license (http://creativecommons.org/licenses/by-nc/4.0/).

\section{INTRODUCTION}

In Statistics, trends are steady and methodical fluctuations in the variance of a process over a long period of time and their underlying features are embedded with useful information that are viable for investment decisions [1-4]. The uncertainty or probabilistic nature of stock investment has made trend analysis a potential and promising tool for investors, who seek capital appreciation, hence, the quest for an appropriate model that could offer the best prediction.

The motivation for this study is drawn from the fact that investment trends are stochastic and follow a Markov process which deals with probability of any particular future behavior of a process with an exactly known current state which cannot be altered by additional knowledge concerning its past behavior [5]. According to [6], Markov chains are fundamental stochastic processes that have many diverse applications and it represents any dynamical system whose new state is simply a function of the last state and an auxiliary random on the past and present only through the present state.

The striking reason for continuous application of Markov chains in investment trend analysis is the existence of a limiting probability distribution which is independent of initial states, and this is associated with the convergence in probability of finding the Markov chain in a particular state irrespective of which state the chain began at time zero.

Prior studies such as [7-10] applied Markov chain model approach to analyze and forecast stock trends. On the other hand, given the fact that Markov chain models are appropriate in tracking long-run behavior of trends but failed in tracking short-term behavior prompted the studies of [11-15] to provide the missing link.

However, no previous studies have attempted to take into consideration the effect of changing variance while analyzing investment trends through Markov chains. It is evident in the literature that stocks trends are characterized by changing variance [16-23]. The consequential effect of neglecting changing variance is that it takes longer time to achieve convergence of the probabilities of Markov chain. For instance, in the study of [9], it takes about 2080 steps (about 8 trading years) to attain near convergence.

Therefore, this study seeks to bridge this gap by stabilizing the changing variance through logarithmic transformation to achieve good stability and quick convergence. This is advantageous in that, it reduces the number of $n$-step computation and increases the precision of the estimates of the probabilities, thus enhances investors to make quick and stable decision.

*Corresponding author. Email: eubong44@gmail.com 
The remaining part of the paper is organized as follows: Section 2 treats materials and methods. Results and discussion are presented in Section 3 while the conclusion is handled in Section 4.

\section{MATERIALS AND METHODS}

\subsection{Markov Process}

A Markov chain $\left\{X_{t}\right\}$ is a stochastic process with the property that, given the value of $X_{t}$, the values of $X_{s}$ for $s>t$ are not influenced by the values of $X_{u}$ for $u<t$. A discrete-time Markov chain is a Markov process whose state space is a finite or countable set, and whose (time) index set is $T=(0,1,2, \ldots)$.

Mathematically, the Markov property is that

$$
\operatorname{Pr}\left\{X_{n+1}=j \mid X_{0}=i_{0}, \cdots, X_{n-1}=i_{n-1}, X_{n}=i\right\}=\operatorname{Pr}\left\{X_{n+1}=j \mid X_{n}=i\right\}
$$

for all time points $\mathrm{n}$ and all states $i_{0}, \ldots, i_{n-1}, i, j[6,5]$.

\subsection{Transition Probability Matrices of a Markov Chain}

The probability of $X_{n+1}$ being in state $j$ given that $X_{n}$ is in state $i$ is called the one-step transition probability and is denoted by $P_{i j}^{n . n+1}$. That is,

$$
P_{i j}^{n \cdot n+1}=\operatorname{Pr}\left\{X_{n+1}=j \mid X_{n}=i\right\}
$$

The notation emphasizes that in general, the transition probabilities are functions not only of the initial and final states, but also of the time of transition as well. When the one-step transition probabilities are independent of the time variable $n$, we say that the Markov chains have stationary transition probabilities. Given the Markov chains that have stationary transition probabilities, then, $P_{i j}^{n . n+1}=P_{i j}$ is independent of $n$, and $P_{i j}$ is the conditional probability that the state value undergoes a transition from $i$ to $j$ in one trial. Thus $P_{i j}$ is arranged in a matrix, in the infinite square array

$$
\mathbf{P}=\left\|\begin{array}{lllll}
\mathbf{P}_{00} & \mathbf{P}_{01} & \mathbf{P}_{02} & \mathbf{P}_{03} & \cdots \\
\mathbf{P}_{10} & \mathbf{P}_{11} & \mathbf{P}_{12} & \mathbf{P}_{13} & \cdots \\
\mathbf{P}_{20} & \mathbf{P}_{21} & \mathbf{P}_{22} & \mathbf{P}_{23} & \cdots \\
\vdots & \vdots & \vdots & \vdots & \\
\mathbf{P}_{10} & \mathbf{P}_{\mathrm{i} 1} & \mathbf{P}_{\mathrm{i} 2} & \mathbf{P}_{\mathrm{i} 3} & \cdots \\
\vdots & \vdots & \vdots & \vdots &
\end{array}\right\|
$$

where $P=\left\|P_{i j}\right\|$ is referred to as the Markov matrix or transition probability matrix of the process. The ith row of $\mathbf{P}$, for $i=0,1, \ldots$, is the probability distribution of the values of $X_{n+1}$ under the condition that $X_{n}=i$. If the number of states is finite, then $P$ is a finite square matrix whose order (the number of rows) is equal to the number of states. Clearly, the quantities $P_{i j}$ satisfy the conditions

$$
\begin{gathered}
P_{i j} \geq 0 \text { for } i, j=0,1,2, \ldots, \\
\sum_{\mathrm{j}=0}^{\infty} P_{i j}=1 \text { for } i=0,1,2, \ldots
\end{gathered}
$$

A Markov process is completely defined once its transition probability matrix and initial state $X_{0}$ (or, more generally, the probability distribution of $\left.X_{0}\right)$ are specified $[5,6]$.

\subsection{Regular Transition Probability Matrices}

Suppose that a transition probability matrix $P=\left\|P_{i j}\right\|$ on a finite number of states labeled $0,1, \ldots, n$ has the property that when raised to some power $n$, the matrix $P^{n}$ has all of its elements strictly positive. Such a transition probability matrix, or the corresponding Markov chain is called regular. The most important fact concerning a regular Markov chain is the existence of limiting probability distribution $\pi=\left(\pi_{0}, \pi_{1}, \ldots, \pi_{n}\right)$, where $\pi_{j}>0$ for $j=0,1, \ldots, n$ and $\sum_{j} \pi_{j}=1$, and this distribution is independent of the initial state. Formally, for a regular transition probability matrix $P=\left\|P_{i j}\right\|$ we have the convergence

$$
\lim _{n \rightarrow \infty} P_{i j}^{(n)}=\pi_{j}>0 \text { for } j=0,1, \ldots, n .
$$


Or, in terms of the Markov chain $\left\{X_{n}\right\}$

$$
\lim _{n \rightarrow \infty} \operatorname{Pr}\left\{X_{n}=j \mid X_{0}=i\right\}=\pi_{j}>0 \text { for } j=0,1, \ldots, n
$$

This convergence means that, in the long run $(n \rightarrow \infty)$, the probability of finding the Markov chain in state $j$ is approximately $\pi_{j}$ no matter in which state the chain began at time zero [5].

\subsection{Proposed Transformation}

In traditional Markov chain process, the problem of estimating transition probabilities is based on the observations on the Markov chain at time $t=\{0,1, \ldots, T\}$ using

$$
n_{i j}=\sum_{t=0}^{T} n_{i j}(t)
$$

where $n_{i j}(t)$ is the number of chain transitions from the state $i$ to the state $j$ at time $t$.

$$
n_{i}=\sum_{j=1}^{k_{i}} n_{i j}
$$

where $n_{i}$ is the total number of chain transitions from the state $i$ over the time $T$ [24,25]. Hence, the estimates of transition probabilities from the state $i$ to state $j$ are obtained as follows:

$$
\hat{P}_{i j}=\frac{n_{i j}}{n_{i}}, i=1, \ldots, k ; j=1, \ldots, k_{i}
$$

Traditional Markov chain is so defined to enhance convergence to a certain probability distribution of interest. Howbeit, to achieve quick convergence rate of the steady state probabilities, this study considers natural logarithmic transformation of the observations on the Markov chain $t=\{0,1, \ldots, T\}$ which is reflected in the following sample statistics:

$$
n_{i j}^{\prime}=\sum_{t=0}^{T} \ln \left(n_{i j}\right)(t)
$$

where $n_{i j}^{\prime}(t)$ is the number of logarithmic chain transitions from the state $i$ to the state $j$ at time $t$.

$$
n_{i j}^{\prime}=\sum_{j=1}^{k_{i}} \ln \left(n_{i j}\right)
$$

where $n_{i}$ is the total number of logarithmic chain transitions from the state $i$ over the time $T$.

$$
\hat{P}_{i j}^{\prime}=\frac{n_{i j}^{\prime}}{n_{i}^{\prime}}, i=1, \ldots, k ; j=1, \ldots, k_{i},
$$

where $\hat{P}_{i j}^{\prime}$ are the estimates of logarithmic transition probabilities from the state $i$ to state $j$. This new approach distinguishes itself from the traditional Markov chain approach by incorporating the natural logarithm for the purpose of accounting for changing variance. Stabilizing the changing variance in turn aids quicker convergence of transition probabilities of the Markov chain leading to significant improvements in the estimates of the steady state probabilities.

\section{RESULTS AND DISCUSSION}

\subsection{Data}

The data on stock prices from 2015 to 2018 were obtained from Nigerian Stock Exchange annual report 2018 and are presented in Table 1 while the logarithmic-transformed stock prices are presented in Table 2. The data are grouped into four phases according to stock market cycles: accumulation phase (where the stocks are oversold); mark-up phase (where the demand for the stocks surpasses the supply); distribution phase (where the stocks are overbought) and mark-down phase (where the supply for the stocks overwhelms the demand).

The plots of data in Tables 1 and 2 are shown in Figures 1 and 2, respectively. It is obvious that the direction of movement of the stock prices according to stock cycles changes due to variance stabilization through natural logarithm as shown in Figure 2 compared to the series in Table 1. 
Table 1 Stock prices (“000,000,000”) from 2015 to 2018 according to stock cycle.

\begin{tabular}{lccccc}
\hline & Accumulation & Mark-up & Distribution & Mark-down & Row Total \\
\hline Accumulation & 478 & 501 & 487 & 421 & 1887 \\
Mark-up & 524 & 523 & 536 & 540 & 2123 \\
Distribution & 605 & 521 & 526 & 507 & 2159 \\
Mark-down & 527 & 536 & 430 & 425 & 1918 \\
Grand Total & & & & & $\mathbf{8 0 8 7}$ \\
\hline
\end{tabular}

Table 2 Natural logarithmic-transformed stock prices (“000,000,000”) from 2015 to 2018 according to stock cycle.

\begin{tabular}{lccccc}
\hline & Accumulation & Mark-up & Distribution & Mark-down & Row Total \\
\hline Accumulation & 6.1696 & 6.2166 & 6.1883 & 6.0426 & 24.6171 \\
Mark-up & 6.2615 & 6.2596 & 6.2841 & 6.2916 & 25.0968 \\
Distribution & 6.4052 & 6.2558 & 6.2653 & 6.2285 & 25.1548 \\
Mark-down & 6.2672 & 6.2841 & 6.0638 & 6.0521 & 24.6672 \\
Grand Total & & & & & $\mathbf{9 9 . 5 3 5 9}$ \\
\hline
\end{tabular}

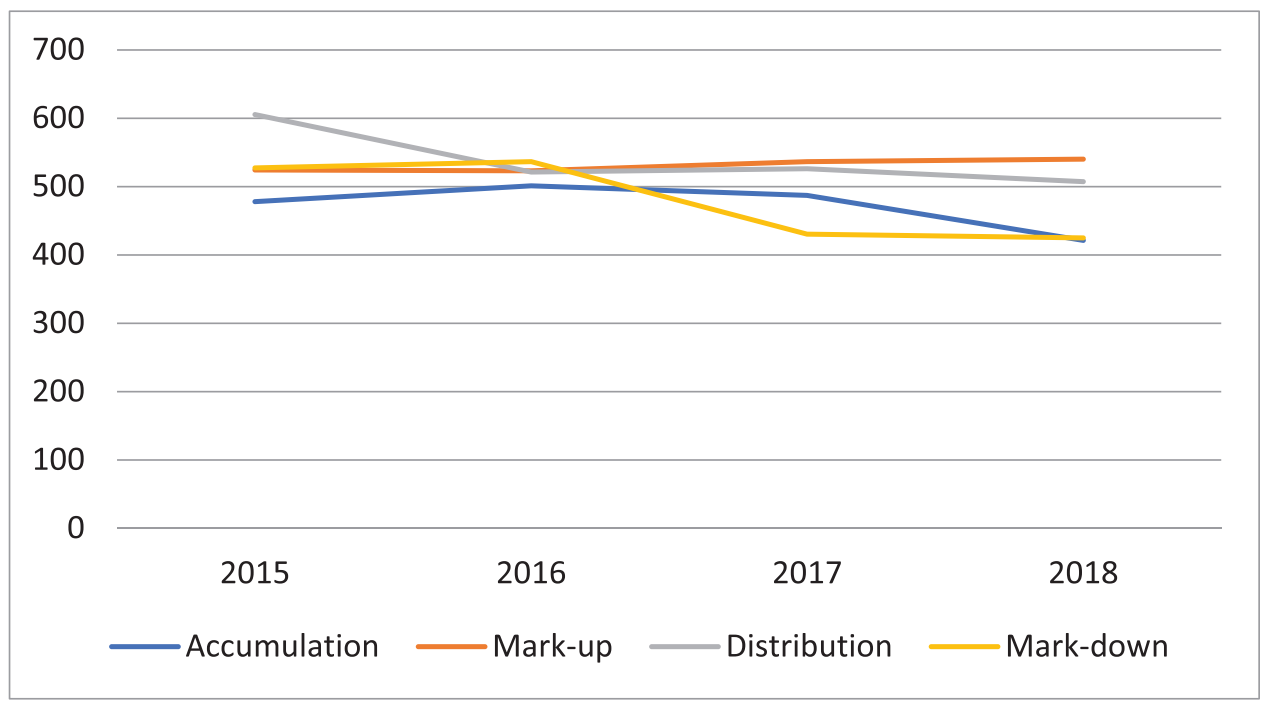

Figure 1 Graph stock prices according to stock cycle.

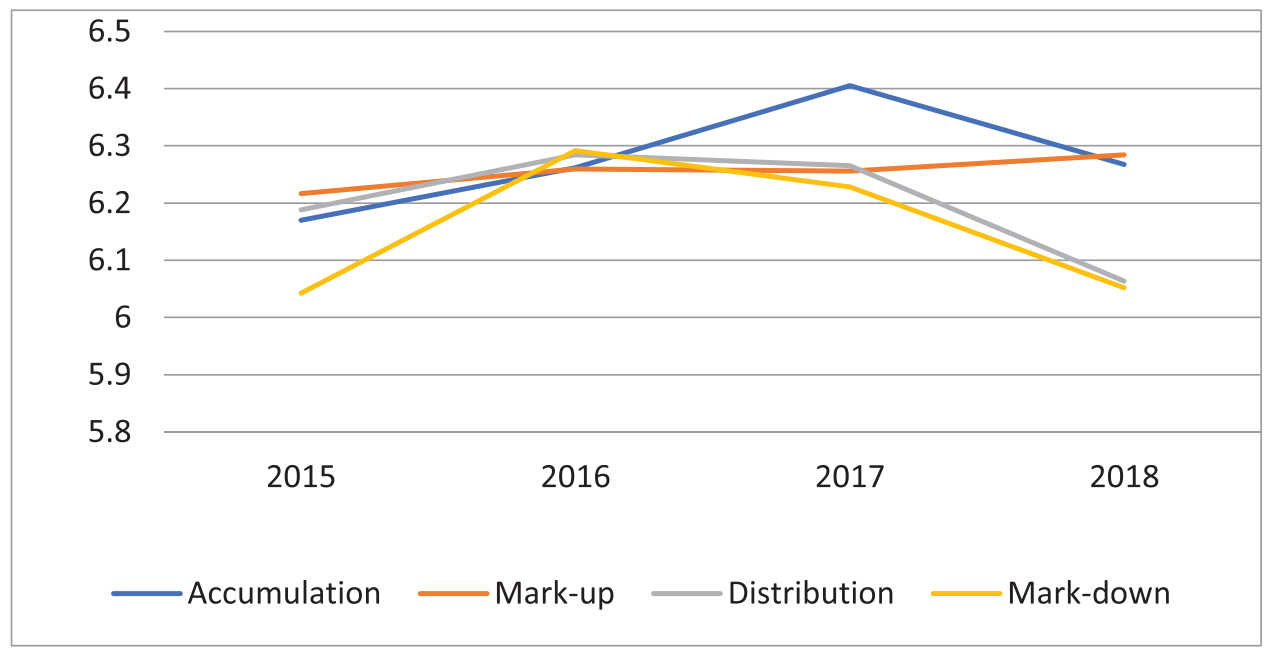

Figure 2 Graph of natural logarithmic-transformed stock prices according to stock cycle. 


\subsection{Building Transition Probability Matrix of the Stock Prices}

The transition probability matrix of the stock cycle using the information in Table 1 are obtained as

\begin{tabular}{llcccc}
\hline & Accumulation & Mark-up & Distribution & Mark-down \\
\hline \multirow{3}{*}{$P_{\text {Stock Cycle }}=$} & Accumulation & 0.2533 & 0.2655 & 0.2581 & 0.2231 \\
& Mark-up & 0.2468 & 0.2463 & 0.2525 & 0.2544 \\
& Distribution & 0.2802 & 0.2413 & 0.2436 & 0.2348 \\
& Mark-down & 0.2748 & 0.2795 & 0.2242 & 0.2216 \\
\hline
\end{tabular}

\subsection{Convergence of Steady State Probability of the Stock Prices}

The steady state probabilities are the constant probabilities to be attained in the states in the future. The interest is to ascertain the number of steps it takes $P_{\text {StockCycle }}$ to attain a steady state.

\begin{tabular}{llcccc}
\hline & & Accumulation & Mark-up & Distribution & Mark-down \\
\hline \multirow{3}{*}{$P_{\text {Stock Cycle }}^{2}=$} & Accumulation & 0.2633 & 0.2573 & 0.2453 & 0.2341 \\
& Mark-up & 0.2640 & 0.2582 & 0.2444 & 0.2334 \\
& Distribution & 0.2633 & 0.2583 & 0.2452 & 0.2331 \\
& Mark-down & 0.2623 & 0.2578 & 0.2458 & 0.2341 \\
\hline
\end{tabular}

\begin{tabular}{llcccc}
\hline & & Accumulation & Mark-up & Distribution & Mark-down \\
\hline \multirow{3}{*}{$P_{\text {Stock Cycle }}^{3}=$} & Accumulation & 0.2633 & 0.2579 & 0.2452 & 0.2337 \\
& Mark-up & 0.2632 & 0.2579 & 0.2452 & 0.2337 \\
& Distribution & 0.2632 & 0.2579 & 0.2452 & 0.2337 \\
& Mark-down & 0.2633 & 0.2579 & 0.2451 & 0.2337 \\
\hline
\end{tabular}

\begin{tabular}{llcccc}
\hline & & Accumulation & Mark-up & Distribution & Mark-down \\
\hline \multirow{3}{*}{$P_{\text {Stock Cycle }}^{4}=$} & Accumulation & 0.2632 & 0.2579 & 0.2452 & 0.2337 \\
& Mark-up & 0.2632 & 0.2579 & 0.2452 & 0.2337 \\
& Distribution & 0.2632 & 0.2579 & 0.2452 & 0.2337 \\
& Mark-down & 0.2632 & 0.2579 & 0.2452 & 0.2337 \\
\hline
\end{tabular}

Therefore, the $P_{\text {StockCycle }}$ is found to converge to a steady state at the forth power.

\subsection{Convergence of Steady State Probability of the Natural Logarithmic-Transformed Stock Prices}

The focus is to determine how fast the logarithmic-transformed transition probability matrix, $P_{\text {Ln(StockCycle) }}$ converges to the steady state. 


\begin{tabular}{llcccc}
\hline & & Accumulation & Mark-up & Distribution & Mark-down \\
\hline$P_{\text {Ln (Stock Cycle })}=$ & Accumulation & 0.2506 & 0.2525 & 0.2514 & 0.2455 \\
& Mark-up & 0.2495 & 0.2494 & 0.2504 & 0.2507 \\
& Distribution & 0.2546 & 0.2487 & 0.2491 & 0.2476 \\
& Mark-down & 0.2541 & 0.2548 & 0.2458 & 0.2454 \\
\hline
\end{tabular}

\begin{tabular}{llcccc}
\hline & & Accumulation & Mark-up & Distribution & Mark-down \\
\hline \multirow{3}{*}{$P_{\text {Ln(Stock Cycle })}^{2}=$} & Accumulation & 0.2522 & 0.2513 & 0.2492 & 0.2473 \\
& Mark-up & 0.2522 & 0.2514 & 0.2492 & 0.2473 \\
& Distribution & 0.2522 & 0.2514 & 0.2492 & 0.2473 \\
& Mark-down & 0.2522 & 0.2513 & 0.2492 & 0.2473 \\
\hline
\end{tabular}

\begin{tabular}{llcccc}
\hline & & Accumulation & Mark-up & Distribution & Mark-down \\
\hline \multirow{3}{*}{$P_{\text {Ln(Stock Cycle })}^{3}=$} & Accumulation & 0.2522 & 0.2513 & 0.2492 & 0.2473 \\
& Mark-up & 0.2522 & 0.2513 & 0.2492 & 0.2473 \\
& Distribution & 0.2522 & 0.2513 & 0.2492 & 0.2473 \\
& Mark-down & 0.2522 & 0.2513 & 0.2492 & 0.2473 \\
\hline
\end{tabular}

It could be found that $P_{\operatorname{Ln}(\text { Stock Cycle) }}$ converges to the third power.

\subsection{Percentage Difference between the Steady State Transition Probability Matrix of Stock Prices and Natural Logarithm of Stock Prices}

As indicated in Table 3, when the variance (variation in the prices) is not accounted for, at the accumulation cycle, the stocks have the probability of 0.2632 , that is, about $26.32 \%$ of being oversold. At the mark-up cycle, the probability of the demand for the stocks surpassing the supply is 0.2579 which is about $25.79 \%$. At the distribution cycle, the probability of the stock being overbought is 0.2452 which is about $24.52 \%$, and at the mark-down cycle, the probability of the supply of stocks overwhelming the demand is $0.2337(23.37 \%)$. On the other hand, when the stabilization of variance is considered, the accumulation cycle has the probability of $0.2522(25.22 \%)$ of the stocks being oversold. At the mark-up cycle, the probability that the demand for stocks is higher than supply is 0.2513 (25.13\%). At distribution cycle, the probability of the stocks being overbought is $0.2492(24.92 \%)$, and at the mark-down cycle, the supply is greater than demand with the probability of $0.2473(24.73 \%)$. Comparing the two approaches, it is shown that, when the logarithmic transformation is not considered, the probabilities that the stocks would remain in the accumulation and mark-up cycles are hyped by $1.1 \%$ and $0.7 \%$, respectively while the probabilities that the stocks remain in distribution and mark-down are played down by $0.41 \%$ and $1.36 \%$, respectively.

Going forward, the logarithmic-modified transition probability matrix converged at third power, a step faster than that of the ordinary transition probability matrix which converged at the fourth power. In addition, the true direction of the stock cycles appeared to be misleading when the changing variance is not accounted for. The findings of this study are in agreement with previous studies of [7-15], and that Markov chain approach is sufficient for modeling stock/investment trends but proud of its novelty by application of logarithmic transformation of the transition matrix and provided the needed improvement to the study of [9]. The implication is that, a quicker convergence with higher precision estimates of the steady state probabilities is achieved when the variance is stabilized.

\section{CONCLUSION}

Concisely, the findings of the study showed that the logarithmic-modified approach to Markov chain modeling of the investment trends results in a faster convergence rate of the steady state transition probability with robust probabilities than the ordinary approach. Thus the aim of this study is sufficed by these findings. The weakness of the study is its inability to assess the expected number of stocks in the long run using logarithmic-modified approach and it is therefore recommended to be undertaken in our future studies.

\section{CONFLICTS OF INTEREST}

The authors declare no conflicts of interest regarding the publication of this paper. 
Table 3 Comparison of the steady state transition probability matrix of stock prices and natural logarithm of stock prices.

\begin{tabular}{lccc}
\hline Stock Cycle & $\begin{array}{c}\text { Steady State Transition Probability Matrix } \\
\text { of Stock Prices }\end{array}$ & $\begin{array}{c}\text { Steady State Transition Probability Matrix } \\
\text { of Natural Logarithm of Stock Prices }\end{array}$ & $\begin{array}{c}\text { Percentage } \\
\text { Differences (\%) }\end{array}$ \\
\hline Accumulation & 0.2632 & 0.2522 & 1.1 \\
Mark-up & 0.2579 & 0.2513 & 0.7 \\
Distribution & 0.2452 & 0.2492 & -0.41 \\
Mark-down & 0.2337 & 0.2473 & -1.36 \\
\hline
\end{tabular}

\section{AUTHORS' CONTRIBUTIONS}

MIU designed the study and wrote the first draft. JAU managed the literature search, manuscript writing and data collection. EAA managed the analysis. All authors read and approved the final manuscript.

\section{DATA AVAILABILITY STATEMENT}

The authors confirm that the data supporting the findings of the study are available within the article.

\section{ACKNOWLEDGMENTS}

The authors received no specific funding for this article.

\section{REFERENCES}

1. I.U. Moffat, E.A. Akpan, Int. J. Adv. Appl. Sci. 4 (2017), 96-100.

2. J.D. Cryer, K.S. Chan, Time Series Analysis with Application in R, second ed., Springer, New York, NY, USA, 2008 , p. 27.

3. W.W.S. Wei, Time Series Analysis Univariate and Multivariate Methods, second ed., Adison Westley, New York, NY, USA, 2006, pp. 69-71.

4. W.A. Fuller, Introduction to Statistical Time Series, second ed., John Wiley and Sons, New York, NY, USA, 1996, pp. 475-484.

5. H.M. Taylor, S. Karlin, An Introduction to Stochastic Modeling, third ed., Academic Press, San Diego, CA, USA, 1998, pp. 96-200.

6. R. Serfozo, Basics of Applied Stochastic Processes, Probability and Its Applications, first ed., Springer-Verlag, Berlin, Heidelber, Germany, 2009, pp. 1-3.

7. A. Fitriyando, T.E. Lestari, Application of Markov chain to stock trend: a study of PTHM Sampoerna tbk, in 3rd Annual Applied Science and Engineering Conference (AASEC 2018) IOP Conference Series: Materials Science and Engineering, Bandung, Indonesia, 2018, vol. 434, p. 012007.

8. M.K. Bhusal, Int. J. Sci. Eng. Res. 8 (2017), 1733-1745.

9. S. Otieno, E.O. Olumuba, R.N. Nyabwanga, Kenya, Int. J. Curr. Res. 7 (2015), 14712-14721.

10. K.J. Doubleday, J. Esunge, J. Math. Stat. 7 (2011), 103-106.

11. Y. Zheng, T. Shu, S. Chen, K.K. Lai, in: C.-K. Ngan (Ed.), Time Series Analysis: Data, Methods, and Applications, IntechOpen, Princes Gate Court, London, SW72QJ, United Kingdom, 2019. https://www.intechopen.com/books/time-series-analysis-data-methods-and-applications/ using-gray-markov-model-and-time-series-model-to-predict-foreign-direct-investment-trend-for-support

12. K. Ganesan, U. Annamalai, N. Deivanayagampillai, Financ. Innov. 5 (2019), 35.

13. K.C. Chan, Int. J. Innov. Comput. Inf. Control. 11 (2015), 1205-1218.

14. M. Bicego, E. Grosso, E. Otranto, in: N. da Vitoria Lobo et al. (Eds.), Structural, Syntactic, and Statistical Pattern Recognition, SSPR/SPR 2008, Lecture Notes in Computer Science, vol. 5342, Springer, Berlin, Heidelberg, Germany, 2008.

15. O. Netzer, J.M. Lattin, V. Srinivasan, Mark. Sci. 27 (2008), 185-204.

16. I.U. Moffat, E.A. Akpan, Int. J. Anal. Appl. 18 (2020), 559-571.

17. D. Berger, I. Dew-Becker, S. Gilglio, Rev. Econ. Stud. 87 (2020), 40-76.

18. B.M. Bein, Sustainability. 12 (2020), 1-14.

19. E.A. Akpan, I.U. Moffat, Int. J. Anal. Appl. 17 (2019), 530-547.

20. I.U. Moffat, E.A. Akpan, Appl. Math. 10 (2019), 989-1003.

21. E.F. Fama, K.R. French, J. Econ. Perspect. 18 (2004), 25-46.

22. Y. Hamao, R.W. Masulis, V. Ng, Rev. Financ. Stud. 3 (1990), 281-307.

23. M.A. King, S. Wadhwani, Rev. Financ. Stud. 3 (1989), 5-33.

24. A. Tesselkin, V. Khabarov, Procedia Eng. 178 (2017), 107-116.

25. F.O. Mettle, E.N. Quaye, R.A. Laryea, SpringerPlus. 3 (2014), 657. 